\title{
Renewed support for surgical safety checklist
}

\author{
Cite as: CMAJ 2017 November 20;189:E1431-2. doi: 10.1503/cmaj.109-5524
}

Posted on cmajnews.com on Oct. 31, 2017.

$\mathbf{N}$ ine years ago, after an unknown mishap, Donna Penner woke up during abdominal surgery. "I was paralyzed and unable to communicate and so I remained awake, fully alert, feeling everything they did to me," she recounts. "It was horrific, absolutely horrific."

During the ordeal, Penner overheard members of the surgical team say that the anesthesiologist had left the operating room without telling anyone where he was going. Although the actual error is unknown in Penner's case, awareness under anesthesia usually occurs because the patient receives an insufficient dose of anesthetic drugs during surgery. That can happen because of equipment failure, error on the part of the anesthetist, or patient factors that mean more anesthetic is required than calculated to be necessary.

Now, Penner is lending her voice to a renewed call for uptake of a modified World Health Organization (WHO) surgical safety checklist. "I'd like to think in my case it would have been helpful."

The Canadian Patient Safety Institute (CPSI) recently issued a position statement urging hospitals to support adoption and use of the checklist as a standard part of every surgical procedure. The statement was cosigned by Alberta Health Services, the Canadian Anesthesiologists' Society and the Operating Room Nurses Association of Canada, and endorsed by half a dozen surgical associations.

"This is a call to action from the people who work in operating rooms every day, who see the benefit," says CPSI CEO Chris Power. "Every 13 minutes, someone dies from a preventable adverse event in Canada, and no other industry would find that acceptable."

The checklist is a series of questions that surgical teams complete together before anesthesia, before incision and before a patient leaves the operating room. Used correctly, it helps teams communicate and avoid "never events" like performing surgery on the wrong body part or leaving surgical instruments inside a patient, Power explains. "Lack of teamwork and poor communication is really the root cause of most errors."

Many hospitals took up the WHO checklist after a 2009 pilot study showed it reduced deaths and surgical complications by a third. But Power says there's been a "cooling off" in recent years. "Some don't use it consistently, some use their own checklist, not the standardized one based on best practice, and some have opted not to use it because they didn't believe it was effective."

Since the pilot study, smaller studies have supported the initial finding linking checklists to reductions in complications and deaths, and shown additional benefits, including reduced costs and an improved safety culture. However, more recent studies of checklist implementa-

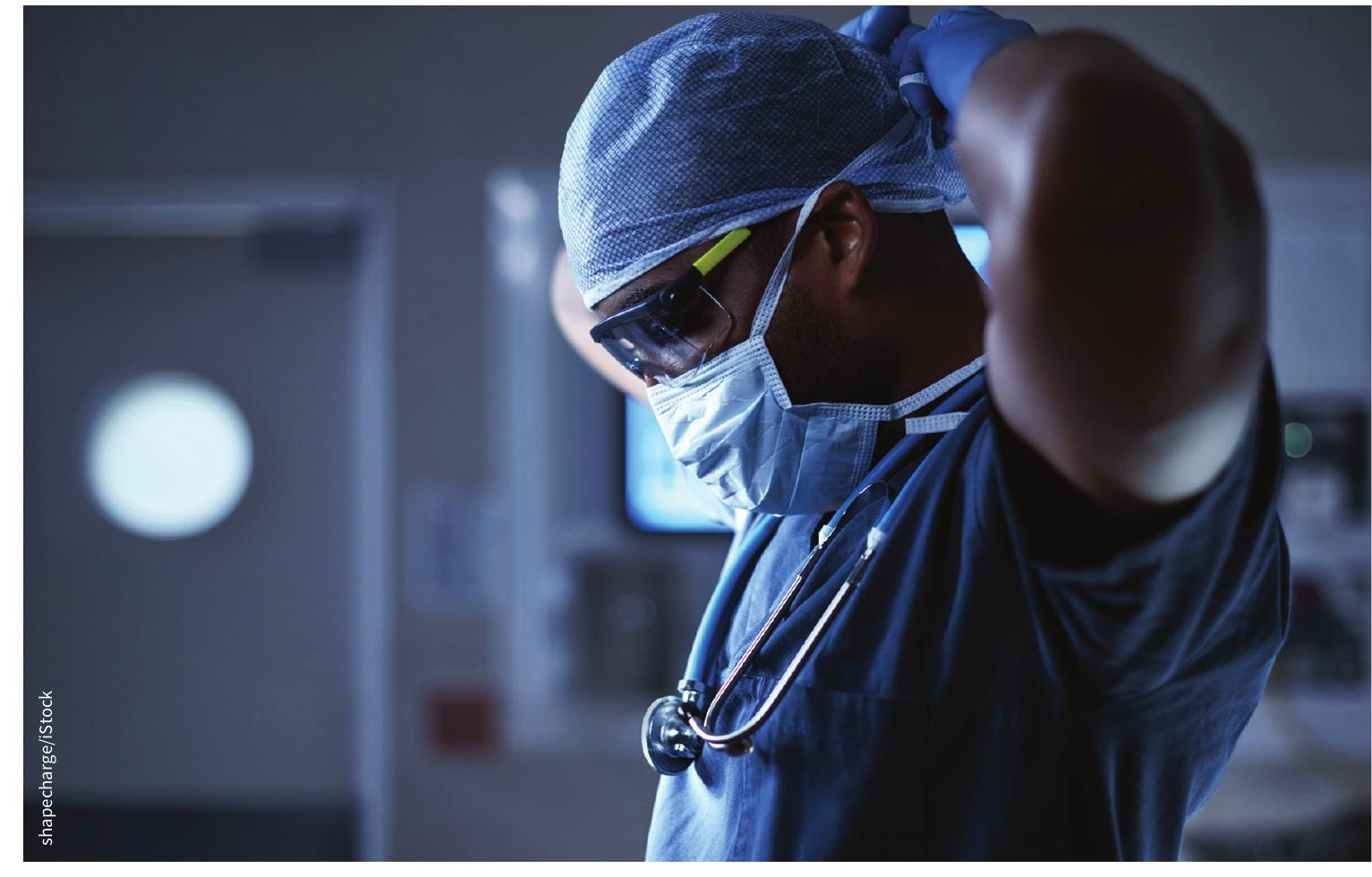

Concerns about the effectiveness of checklists to prevent surgical errors are unfounded, say patient safety advocates. 
tion in Ontario published in the New England Journal of Medicine (NEJM) and CMAJ showed no meaningful improvements in patient safety.

Power argues this doesn't mean the checklists were ineffective. "Many people weren't using them appropriately or were checking them off at the end of the surgery."

Convincing health workers to buy into change is often a sticking point in patient safety, Power says. "You hear a lot about quality improvement being seen as something that comes from on high, rather than from the people who are involved in the day to day."

Training and ensuring that surgical teams see the tangible benefits of using checklists are part of creating an environment that is "conducive to effective adoption," she explains. "We know those organizations who are using it and who have implemented it in a way that people were really engaged, that incidents were reduced significantly by using the checklist."

She points to Alberta Health Services as an example of a health system that is "really working hard to make this part of their culture in the operating room." In 2014-15, "good catches" or errors averted were reported in $4 \%$ of cases as a result of the checklist, and that number has since fallen below $2 \%$.

"One person unnecessarily harmed is too many, and if we know through evidence and best practice that checklists work and help prevent patient safety incidents, then shame on us if we don't use them," says Power. "There's no excuse."

Lauren Vogel, CMAJ 